\begin{tabular}{|l|l} 
REVISTA & $\begin{array}{l}\text { Revista Educación } \\
\text { ISSN: 0379-7082 } \\
\text { ISSN: 2215-2644 } \\
\text { revedu@ gmail.com } \\
\text { Universidad de Costa Rica } \\
\text { Costa Rica }\end{array}$
\end{tabular}

\title{
Incidencia de las prácticas tempranas en la formación inicial del profesorado de educación básica
}

Castro- Rubilar, Juana Irene; Navas - Martinez, Leandro

Incidencia de las prácticas tempranas en la formación inicial del profesorado de educación básica

Revista Educación, vol. 42, núm. 1, 2018

Universidad de Costa Rica, Costa Rica

Disponible en: http://www.redalyc.org/articulo.oa?id=44051918013

DOI: https://dx.doi.org/10.15517/revedu.v42i1.22963

Esta obra está bajo una Licencia Creative Commons Atribución-NoComercial-SinDerivar 3.0 Internacional. 


\title{
Incidencia de las prácticas tempranas en la formación inicial del profesorado de educación básica
}

\author{
The Incidence of Early Internships in Elementary Teacher's Initial Formation
}

Juana Irene Castro-Rubilar [1]

DOI: https://dx.doi.org/10.15517/revedu.v42i1.22963

Universidad Del Bio- Bio, Chile

Redalyc: http://www.redalyc.org/articulo.oa?id=44051918013

jcastro@ubiobio.cl

Leandro Navas - Martinez [2]

Universidad de Alicante, España

Leandro.navas@ua.es

\section{RESUMEN:}

Este estudio es parte de una tesis doctoral que busca, entre otros fines, valorar la percepción que grupos de estudiantes en prácticas tempranas tienen de los aprendizajes logrados en los talleres de lenguaje y matemática, de acuerdo con las competencias específicas del perfil de egreso de la carrera de Pedagogía en Educación General Básica de la Universidad del Bío-Bío. La investigación asume el enfoque cuantitativo y considera un diseño de tipo no-experimental. Participantes: 164 estudiantes del séptimo semestre de la carrera, que realizan sus prácticas en dos tipos de establecimientos: municipalizados (públicos) y particular subvencionados. Responden un cuestionario tipo Likert elaborado ad hoc basado en los indicadores extraídos de las competencias del perfil de egreso. Se llevan a cabo análisis factoriales y correlacionales. Tras el análisis de los resultados, se puede señalar que el estudiantado en prácticas logra aprendizajes vinculados esencialmente a los contenidos disciplinarios que debe enseñar; sin embargo, se observa que el estudiantado en prácticas no tiene autonomía para planificar ni evaluar los aprendizajes que deben lograr. También se evidencia que las metas de aprendizajes, para el alumnado que atenderá durante sus prácticas, no son fijadas por los futuros o futuras docentes. Hallazgo este último que debería llevar a definir competencias más específicas y pertinentes que permitan desarrollar prácticas tempranas que reditúen el perfil de egreso, considerando la vinculación a las comunidades escolares como una oportunidad de aprendizajes pedagógicos.

Palabras Clave: formación profesional, prácticas, aprendizajes activos, desarrollo de habilidades, estudiante de prácticas.

\section{ABstract:}

This study is part of a doctoral thesis that aimed, among other purposes, to value the perception that students in early internships have from achieved learning outcomes in the language and mathematics workshops, according to specific competences from a graduate profile of general elementary education career of UBB. The research has a quantitative approach and consider a nonexperimental type design. The guiding question for the study is focus towards the perception that fourth graders of PEGB, have of achieved learning outcomes in language and math practice workshops in relation to the specific competences of graduate profile in the initial teacher training. The participants are 164 students from seventh semester of the career that do their practice in two types of schools, preferentially public and subsidized. They answer a likert type questionnaire worked out ad hoc and which is based in the extracted indicators of graduate profile competences. Factorial and correlational analysis are carried out. The results analysis, it can be said that the students, in practice period, achieve learning outcomes, linked essentially with disciplinary contents that they have to teach, however, it is observed that they have no autonomy to plan neither to evaluate the learning outcomes that they have to reach. Learning goals for the students, that they will assist during the practice period, are not set by themselves. This last finding

\section{NotAS DE AUTOR}

[1] Doctora en Educación e Investigación Educativa: Enseñanza y Aprendizaje, Universidad de Alicante, España. Directora provincial de Educación Nuble, Chile (Ministerio de Educación 2000-2010). Actualmente se desempeña como académica de jornada completa del Departamento de Educación Universidad Del Bío- Bío, Chillán, Chile. Algunas de sus publicaciones son: Ossa, C., Castro, F., Castañeda, M.T. y Castro, J.(2014) Cultura y liderazgo escolar: factores claves para el desarrollo de la inclusión educativa Actualidades Investigativas en Educación. Indizada en REDALYC, SCIELO; Castro, F. y Castro J. (2013) Manual para el Diseño de Proyectos de Gestión Educacional, Propiedad Intelectual N.²31624 otorgado por la DIBAM.

[2] Doctor en Psicología por la Universidad Nacional de Educación a Distancia (España), Profesor Titular de Universidad en el Departamento de Psicología Evolutiva y Didáctica, Facultad de Educación de la Universidad de Alicante (España) y cuyas líneas de investigación giran en torno a la motivación general y aplicada a diversos ámbitos específicos, la evaluación educativa, el rendimiento académico y la atención a las dificultades de aprendizaje y a las necesidades educativas especiales. 
should guide to define more specific and pertinent competences that could allow early practices that readjust the graduate profile, considering the connection with the school communities as an opportunity of pedagogic learning.

KEYWORDS: professional training, practice, active Learning, skills development, student internship.

\section{AnTECEDENTES}

La formación inicial de docentes en Chile ha significado, en los últimos 20 años, entre otras estrategias, el establecimiento de un conjunto de estándares (Ministerio de Educación, 2012) tanto para la formación pedagógica, como disciplinar. Al mismo tiempo, ha contribuido al cambio de escenario para la formación de profesorado, la promulgación de la Ley General de Educación (Ministerio de Educación, 2009), que fija objetivos de aprendizajes (OA) para los niveles de educación parvularia, básica y media. En ambos documentos legales, se explicitan las competencias que el personal docente deben lograr durante su formación, para el efectivo aprendizaje de quienes serán sus alumnos.

En consecuencia, ello obliga a que la formación inicial se resitúe en un contexto de permanente cambio. Son, precisamente, en estos escenarios cambiantes en los que emergen tanto los estándares para la formación inicial docente, como las nuevas normativas legales transversales en el sistema educativo nacional, a los cuales se suman los procesos de acreditación convocados por el Ministerio de Educación a través la Comisión Nacional de Acreditación de Pregrado (CNA). Esto lleva, en el año 2004, a la Facultad de Educación y Humanidades de la Universidad del Bío-Bío a plantearse el rediseño de los planes de estudios y mallas curriculares, para que estas respondan a los requerimientos de las acreditaciones voluntarias a las que concurrió la institución, donde la carrera Pedagogía en Educación General Básica es la primera en asumir tal desafío.

De acuerdo con estas actualizaciones curriculares y, particularmente, la establecida mediante el Decreto N.2.459 (Universidad del Bíobío, 2005), el estudiantado, desde el segundo semestre de la carrera, realiza prácticas tempranas, que en la malla curricular se denominan "Talleres de Práctica”. Esta inclusión en la malla curricular de prácticas tempranas en Lenguaje, Matemática, Comprensión del Medio Natural y Social, Expresión Artística, Educación Física y Tecnología, más el Taller de Conocimiento del Entorno se realiza con el propósito de progresar en el estudio de estas disciplinas y profundizar en su didáctica como lo expresan los "principios básicos" definidos para el efecto. Específicamente, la reforma curricular del área pedagógica definió el área de formación práctica como: "Actividad curricular de habilitación gradual, sistemática y progresiva del estudiante para desempeñar la función docente, de aptitud y complejidad creciente, partiendo de la observación hasta la asunción integral de la práctica con responsabilidad total" (Universidad del Bíobío, 2009, p. 43).

En este sentido, en la formación inicial de docentes en las universidades y, en particular, en la Carrera de Pedagogía en Educación General Básica, se van incorporando propuestas, por ejemplo, las emanadas de la Comisión sobre Formación Inicial Docente (Ministerio de Educación, 2005), en las que se establecen requerimientos de las prácticas para estudiantes de pedagogía:

Las prácticas de los estudiantes de pedagogía en los distintos momentos de su carrera son un elemento central de su proceso formativo y constituyen un eje articulador de la especialidad y la formación pedagógica. Del mismo modo, encausan la relación Escuela - Universidad, por lo que es necesario, por parte de las instituciones formadoras, atender preferentemente la gestión de su supervisión, considerando un tiempo adecuado para esta tarea y el reconocimiento académico a los profesores guía de las instituciones escolares. (Ministerio de Educación, 2005, p.73) 


\section{MARCO TEÓRICO}

En el sistema educativo, en todos sus niveles, desde la publicación del informe de la UNESCO, realizado por la Comisión Internacional sobre Educación, presidida por Delors en 1996, se introducen las competencias en cuatro líneas fundamentales:

Aprender a ser; o actuar con autonomía, juicio y responsabilidad personal. Aprender a conocer; o asimilar conocimientos científicos y culturales generales y específicos, que se complementarán a lo largo de toda la vida. Aprender a hacer, o adquirir procedimientos que ayuden a afrontar las dificultades que se presenten en la vida y en la profesión.

Aprender a ser, a convivir y a trabajar juntos, o comprender mejor a los demás, el mundo y sus interrelaciones. (De Acedo, 2010, p. 16)

De esta manera las competencias, como nuevo paradigma educativo, suponen nuevas formas de diseñar, desarrollar y evaluar la formación en los centros de educación superior (Tejada y Ruíz, 2016), al tiempo que contienen un conjunto de funciones que se van tratando articuladamente en el proceso formativo y estas dan origen a principios sobre los cuales cada vez existe mayor compromiso. Tobón, Prieto y Fraile (2010) los resumen como se muestran en la Tabla 1.

TABLA 1

Principios con mayor consenso en el modelo por competencias

\begin{tabular}{ll}
\hline Pertinencia & $\begin{array}{l}\text { Las instituciones educativas deben generar sus } \\
\text { propuestas de formación articulando su visión y } \\
\text { filosofia con los retos del contexto y las politicas } \\
\text { educativas vigentes. }\end{array}$ \\
\hline Calidad & $\begin{array}{l}\text { Los procesos educativos deben asegurar la } \\
\text { calidad del aprendizaje en correspondencia con } \\
\text { un determinado perfil de formación, } \\
\text { considerando la participación de la comunidad. }\end{array}$ \\
\hline Formar & $\begin{array}{l}\text { Los maestros y maestras deben orientar sus } \\
\text { acciones a formar competencias y no a enseñar } \\
\text { contenidos, los cuales deben ser solo medios. }\end{array}$ \\
\hline \multirow{2}{*}{ Papel docente } & $\begin{array}{l}\text { Los maestros y las maestras deben ser, ante } \\
\text { todo guias, dinamizadores y mediadores, para }\end{array}$ \\
& $\begin{array}{l}\text { competencias. No deben ser solo trasmisores de } \\
\text { contenidos. }\end{array}$ \\
\hline Esencia de las & $\begin{array}{l}\text { Las competencias son actuaciones o } \\
\text { desempeños ante actividades y situaciones } \\
\text { competencias }\end{array}$ \\
& $\begin{array}{l}\text { cotidianas que articulan y movilizan recursos } \\
\text { personales y del contexto externo. }\end{array}$ \\
\hline
\end{tabular}

Nota: Adaptado de Tobón et al. (2010, p. 6).

Los logros de aprendizaje en la formación inicial docente están influidos por factores que se derivan de las características de los programas de formación, del futuro profesorado y del personal formador de este (Ávalos, 2007). En este sentido, la oportunidad de aprender está contenida en el currículo que fue expuesto en el proceso de formación y la forma en la que este fue presentado, incluyendo las actividades de aprendizaje requeridas, "comprende también las experiencias de aprendizaje práctico en contextos educacionales escolares, sean directas o referidas a contextos” (Ávalos y Matus, 2010, p. 27).

Independiente de la orientación que tomen los aprendizajes y las competencias, tiene especial significado plantearse también la contribución que estas hacen al perfil de egreso, como expresión de los procesos formadores que atesoran los aprendizajes logrados en el transcurso de la formación profesional. Se recogen 
de manera sucinta en la Tabla 2 las realizaciones profesionales que Tejada (2011) visualiza como funciones que corresponden al personal docente.

TABLA 2

Realizaciones profesionales

\begin{tabular}{l|l}
\hline Unidad de competencia & Realizaciones profesionales \\
\hline Proporcionar & Crear las óptimas condiciones de \\
oportunidades de & aprendizaje, enfatizando los \\
aprendizaje adaptadas a & aspectos más importantes por \\
las características de los & desarrollar. \\
individuos o grupos y a & Impartir los contenidos formativos \\
Sus necesidades de & del programa utilizando y \\
cualificación, así como & alternando técnicas, estrategias, \\
acompañar y orientar, de & recursos y materiales didácticos. \\
manera contextualizada, \\
Su proceso de aprendizaje & Proponer, dinamizar y supervisar \\
y su cualificación. & actividades de aprendizaje. \\
\hline
\end{tabular}

Nota: Adaptado de Tejada (2011, p. 10).

\section{REFERENTES CONCEPTUALES}

Las competencias enunciadas en los perfiles profesionales y de egreso pueden ser representativas de un conjunto de aprendizajes que, puestos en situación, trascienden un enfoque simple. Así lo destaca Le Boterf (2001) cuando formula:

Una persona competente es una persona que sabe actuar de manera pertinente en un contexto particular eligiendo y movilizando un equipamiento doble de recursos: recursos personales (conocimiento, saber hacer, cualidades, cultura, recursos emocionales, etc.) y recursos de redes (banco de datos, redes documentales, redes de experiencia especializada, etcétera). (p. 54)

La carrera de Pedagogía en Educación General Básica concentra las competencias del perfil de egreso en dos tipos: genéricas y específicas. Las genéricas tienen que ver, fundamentalmente, con los desafíos que señalan los estándares para la formación inicial docente desde el año 2005 y que enfatizan la capacidad de análisis crítico, de investigación - acción y las tareas propias del personal docente. Por otro lado, las competencias específicas apuntan al dominio disciplinario y pedagógico que el futuro personal docente debe tener:

El egresado de la Carrera de PEGB, es un profesional competente que demuestra dominio de los contenidos pedagógicos, como también, de las especialidades correspondientes al curriculum de Educación Básica del sistema educacional vigente. Su temprana vinculación con el medio escolar, urbano y rural, constituye una impronta, que le permite un desarrollo integral, así como un posicionamiento en los distintos escenarios sociales y educativos del país. (Universidad del Bío- Bío, 2009, p. 40)

Las competencias genéricas del perfil de egreso buscan que el estudiantado desarrolle: la capacidad de analizar críticamente el trabajo programático y de aula, la investigación - acción y la capacidad de trabajo en equipo y la toma de decisiones pedagógicas en el ámbito escolar (Universidad del Bíobío, 2009). Las competencias específicas son enumeradas en la Tabla 3 (p. 41).

En el presente estudio, que parte de una tesis doctoral, es referente para la técnica de autoinforme, cuál es el logro de aprendizajes de estudiantes en prácticas y cómo estos grupos tributan al perfil de egreso.

El objetivo general de este estudio es caracterizar el nivel de aprendizajes logrado en las Prácticas de Lenguaje y Matemática de estudiantes de cuarto año de Pedagogía en Educación General Básica, en atención a las competencias específicas del perfil de egreso de la carrera, según su propia opinión. 
La hipótesis de partida es que la realización de los talleres de las prácticas tempranas del séptimo semestre, por parte de estudiantes de la carrera de Pedagogía en Educación General Básica, logra aprendizajes definidos en las competencias específicas en el perfil de egreso. También, que los contenidos y las experiencias desarrolladas en los talleres de las prácticas tempranas, séptimo semestre, contribuyen al logro de aprendizajes establecidos en las competencias del perfil de egreso de la carrera.

\section{MÉTodo}

\section{Participantes}

El procedimiento de muestreo fue incidental, participan en el estudio 164 estudiantes de PEGB de séptimo semestre de la carrera, durante los tres primeros meses del periodo. El $82 \%$ son mujeres y el $18 \%$ hombres. Sus edades están comprendidas entre 20 y 43 años $(M=23$; DT $=5.77)$.

Los nueve centros educativos en los que el estudiantado realizó las prácticas pertenecen al sistema municipal, que representa la educación pública de Chile, y particular subvencionado. La mayor parte de su población escolar se ubica en los niveles socioeconómicos medio bajo y bajo, estratificación otorgada por la aplicación de la prueba estandarizada para medir la calidad de la educación SIMCE. Solo tres establecimientos son de dotación completa, es decir, cuentan con los tres niveles de enseñanza: educación parvularia, educación básica y educación media.

\section{Instrumento}

Se emplea un cuestionario de 30 ítems en sentido positivo (con enunciados favorables) elaborado expresamente para este estudio. Para responder, los sujetos participantes debían indicar su nivel de observación de lo planteado en el texto del ítem en una escala tipo Likert de cinco puntos, cuya graduación es "Siempre”, cuando la acción es observada completamente, "Casi siempre”, cuando la acción es observada con alguna nitidez, "A veces”, cuando la acción es visualizada parcialmente, "Casi nunca”, cuando la acción no se observa plenamente, y "Nunca”, cuando la acción no es observada.

En la elaboración del cuestionario se tuvieron en cuenta las competencias específicas del perfil de egreso, de las que por un análisis lógico - racional se extrajeron una serie de indicadores. En la Tabla 3 se muestra la relación entre competencias, elementos del cuestionario e indicadores.

\section{Procedimiento}

Para llevar a cabo esta investigación se le solicitó apoyo al estudiantado y se dialogó con este en las cuatro secciones de las cuales formaba parte, explicándole y dándole a conocer los objetivos y alcances del estudio. Antes de la aplicación del instrumento se le informó que su participación en la investigación no sería retribuida económicamente, era voluntaria y los datos serían tratados anónimamente. Los cuestionarios fueron aplicados en las respectivas aulas en el periodo de clases normales y en espacios de tiempo de entre 30 a 40 minutos.

\section{Diseño y análisis de datos}

La investigación (Castro, 2015) asume el enfoque cuantitativo y considera un diseño de tipo no experimental, porque no se manipularán las variables y, a su vez, es transversal, ya que se hará la valoración de dichos 
indicadores en un único momento (la aplicación del instrumento). El diseño, al no seleccionarse los sujetos aleatoriamente y al no haber una manipulación intencional de las variables, se ajusta a las características del diseño correlacional ex post facto. Los análisis de los datos se llevan a cabo por medio de técnicas estadísticas descriptivas, factoriales y correlacionales.

TABLA 3

Competencias, elementos del cuestionario e indicadores

\begin{tabular}{|c|c|c|}
\hline Competencias especificas & Estudiantes & Indicadores \\
\hline $\begin{array}{l}\text { Demuestra un dominio de los contenidos disciplinarios de } \\
\text { la especialidad y en la construcción del saber, en diferentes } \\
\text { contextos y situaciones educacionales. }\end{array}$ & $\begin{array}{l}\text { 1- } 2-4-6-7- \\
8-24\end{array}$ & $\begin{array}{l}\text { Dominio de contenidos } \\
\text { disciplinarios en contexto } \\
\text { de aprendizaje en la } \\
\text { escuela. }\end{array}$ \\
\hline $\begin{array}{l}\text { Determina, con claridad y coherencia, las metas de } \\
\text { aprendizaje de sus estudiantes, en especial referidas a } \\
\text { la formación y desarrollo de las competencias de lectura, } \\
\text { escritura y matemáticas en diversas situaciones educativas. }\end{array}$ & 9- $12-13-14$ & $\begin{array}{l}\text { Formulación de metas de } \\
\text { aprendizaje coherentes } \\
\text { con la realidad escolar. }\end{array}$ \\
\hline $\begin{array}{l}\text { Evalúa, por criterios, los procesos de aprendizaje de } \\
\text { los distintos subsectores, de acuerdo con los principios } \\
\text { educacionales del marco curricular vigente y, en } \\
\text { conformidad con un marco de referencia y con instrumentos } \\
\text { correspondientes a la evaluación auténtica. }\end{array}$ & $\begin{array}{l}\text { 10- } 11-23-25- \\
\text { 26- } 27-28-29- \\
30\end{array}$ & $\begin{array}{l}\text { Evaluación de los } \\
\text { aprendizajes de ambas } \\
\text { asignaturas. }\end{array}$ \\
\hline $\begin{array}{l}\text { Adopta una actitud crítica y proactiva de su propio proceso } \\
\text { de enseñanza, autoevaluando el quehacer pedagógico con una } \\
\text { visión holística e integradora para el mejoramiento de los } \\
\text { aprendizajes del estudiantado. }\end{array}$ & $\begin{array}{l}5-16-17-19- \\
20\end{array}$ & $\begin{array}{l}\text { Detección de problemas } \\
\text { didácticos en la } \\
\text { implementación de } \\
\text { contenidos disciplinarios }\end{array}$ \\
\hline $\begin{array}{l}\text { Actúa con autonomía en las decisiones pedagógicas, } \\
\text { respetando las normas generales preestablecidas y } \\
\text { relacionándose de manera efectiva y cordial con la totalidad de } \\
\text { miembros del ámbito escolar en que se desempeña. }\end{array}$ & 15 & $\begin{array}{l}\text { Trabajo en equipo para } \\
\text { la toma de decisiones } \\
\text { pedagógicas }\end{array}$ \\
\hline
\end{tabular}

Nota: elaboración propia.

\section{Resultados}

\section{Análisis descriptivos}

En la Tabla 4 se presentan, en orden decreciente, los 10 ítems con mayor frecuencia relativa en Siempre. En dicha tabla se destaca que el $52.4 \%$ consideró que Siempre "En el periodo de observación participante pudo tomar conocimiento de los contenidos que se estaban tratando en el curso" (ítem 6). Al respecto, en 2012, el Centro de Perfeccionamiento e Investigaciones Pedagógicas (CPEIP) del Ministerio de Educación entregó los estándares para estudiantado egresado de las carreras de PEGB, en los que especifica que la primera formación de docentes debe preocuparse porque estos grupos docentes sean profundos conocedores de las disciplinas que enseñarán. En este sentido, los resultados muestran que casi el 74\% (Siempre y Casi siempre) de estudiantes, antes de comenzar sus prácticas, toma conocimiento de los contenidos disciplinarios que debe enseñar. Asimismo, entre otros, es llamativo que en el ítem 7 ("El profesor o profesora guía del curso le informó de los contenidos disciplinarios que debía abordar”), solo el $41 \%$ opine que siempre fue informado de los contenidos disciplinarios por abordar durante sus prácticas. 
TABLA 4

Primeros 10 lugares según la frecuencia relativa por ítems

\begin{tabular}{|c|c|c|c|c|c|c|}
\hline N. ${ }^{\circ}$ & Ítems & Nunca & $\begin{array}{c}\text { Casi } \\
\text { nunca }\end{array}$ & $\begin{array}{c}\text { A } \\
\text { veces }\end{array}$ & $\begin{array}{c}\text { Casi } \\
\text { siempre }\end{array}$ & Siempre \\
\hline $1^{\circ}$ & $\begin{array}{l}\text { 6. En el periodo de observación participante pudo } \\
\text { tomar conocimiento de los contenidos que se } \\
\text { estaban tratando en el curso. }\end{array}$ & 1.8 & 5.5 & 18.9 & 21.3 & 52.4 \\
\hline $2^{\circ}$ & $\begin{array}{l}\text { 7. El profesor o profesora guía del curso le } \\
\text { informó de los contenidos disciplinarios que debía } \\
\text { abordar. }\end{array}$ & 3.7 & 6.1 & 15.9 & 33.5 & 40.9 \\
\hline $3^{\circ}$ & $\begin{array}{l}\text { 1. Al inicio de sus prácticas recibió información } \\
\text { respecto de los contenidos que le correspondía } \\
\text { desarrollar en el curso. }\end{array}$ & 4.3 & 7.9 & 17.7 & 31.1 & 39 \\
\hline $4^{\circ}$ & $\begin{array}{l}\text { 22. Las estrategias utilizadas favorecieron los } \\
\text { aprendizajes. }\end{array}$ & 0.6 & 6.7 & 16.5 & 41.5 & 34.8 \\
\hline $5^{\circ}$ & $\begin{array}{l}\text { 23. En la planificación curricular se explicitó el } \\
\text { tipo de evaluación que se emplearía. }\end{array}$ & 4.3 & 8.5 & 23.2 & 30.5 & 33.5 \\
\hline $6^{\circ}$ & $\begin{array}{l}\text { 20. La escuela le proporcionó los recursos } \\
\text { necesarios para llevar a cabo estrategias de } \\
\text { aprendizaje. }\end{array}$ & 2.4 & 7.3 & 29.3 & 32.3 & 28.7 \\
\hline $7^{\circ}$ & $\begin{array}{l}\text { 30. Los instrumentos aplicados para evaluar los } \\
\text { aprendizajes fueron efectivos para mostrar los } \\
\text { logros. }\end{array}$ & 1.8 & 7.3 & 24.4 & 37.8 & 28.7 \\
\hline $8^{\circ}$ & $\begin{array}{l}\text { 3. Los contenidos que abordó en las prácticas } \\
\text { fueron tratados en el periodo de formación de la } \\
\text { respectiva especialidad. }\end{array}$ & 1.2 & 5.5 & 28.7 & 36.6 & 28 \\
\hline $9^{\circ}$ & $\begin{array}{l}\text { 18. Las estrategias de aprendizaje se adecuaron a } \\
\text { la realidad del curso. }\end{array}$ & 0.6 & 7.3 & 19.5 & 44.5 & 28 \\
\hline $10^{\circ}$ & $\begin{array}{l}\text { 2. Al inicio de sus prácticas recibió información } \\
\text { respecto a las metodologías utilizadas, proyectos } \\
\text { y programas que se estaban ejecutando en el } \\
\text { curso que le correspondió. }\end{array}$ & 5.5 & 10.4 & 24.4 & 329 & 26.8 \\
\hline
\end{tabular}

Nota: elaboración propia.

\section{Análisis factorial exploratorio y análisis de fiabilidad}

Con las respuestas de estudiantes se realizan análisis factoriales y de fiabilidad empleando como método de extracción el de componentes principales y como método de rotación el de normalización Varimax con Kaiser. En el análisis de fiabilidad se considera el coeficiente de consistencia interna alfa de Cronbach. El ítem 8 "Los contenidos informados por el profesor o profesora son los mismos que usted había estudiado en la universidad" se elimina de la escala, debido a que a este elemento no respondió ni el $50 \%$ de las personas encuestadas.

El índice KMO de medida de adecuación muestral obtenido es 0.79 y la prueba de esfericidad de Bartlett resulta estadísticamente significativa $\left(\chi^{2}=1820.06 ; \mathrm{gl}=406 ; \mathrm{p}<.001\right)$. Esto indica la pertinencia de la realización del análisis factorial. De este análisis surgen siete factores que, en total, dan cuenta del $60.54 \%$ de la varianza explicada. Son los siguientes: "Contenidos de las prácticas y aplicación de estrategias de aprendizaje" (Factor 1); "Conocimiento y aplicación de instrumentos de evaluación" (Factor 2); "Diagnóstico y establecimiento de metas de aprendizaje" (Factor 3); "Metas de aprendizajes establecidas en 
conjunto con el establecimiento (profesorado)" (Factor 4); "Conocimientos de los contenidos disciplinarios (lenguaje y matemática)" (Factor 5); "Requerimientos de recursos didácticos para el aprendizaje” (Factor 6), y "Entrega de información sobre programas existentes" (Factor 7). La estructura factorial obtenida se muestra en la Tabla 5. La fiabilidad para el total de la escala (coeficiente alfa de Cronbach) es 0.90 y el índice de discriminación o de homogeneidad (entendido como el coeficiente de correlación $r$ de Pearson entre la puntuación total de la escala sin considerar el elemento y la puntuación del elemento) promedio es ri-total corregida $=0.46$, lo que significa que la escala tiene un adecuado nivel de consistencia interna y una capacidad de discriminación intermedia.

Como se puede apreciar en la mencionada tabla todos los factores obtenidos, exceptuando el factor 7, presentan adecuados índices de consistencia interna y de discriminación. A pesar de que se podría cuestionar la consistencia interna y la capacidad de discriminación del factor 7 ("Entrega de información sobre programas existentes"), dado que solo saturan en él tres elementos, podría eliminarse, no obstante, considerando la relevancia teórica que puede presentar, a tenor de lo expresado en el marco teórico, se decide mantenerlo. 
TABLA 5

Matriz factorial rotada del análisis factorial exploratorio

\begin{tabular}{l} 
Ítem \\
\hline 1. Al inicio de sus prácticas recibió información respecto de los contenidos que le \\
correspondía desarrollar en el curso. \\
3. Los contenidos que abordó en las prácticas fueron tratados en el periodo de \\
formación de la respectiva especialidad. \\
5. En los talleres de prácticas de las disciplinas considera que profundizó los \\
contenidos de cada especialidad.
\end{tabular}
contenidos de cada especialidad.

16. Las estrategias de aprendizaje fueron diseñadas en el trabajo de talleres en la universidad.

17. Las estrategias de aprendizaje diseñadas fueron apropiadas para los resultados que esperaba del curso.

18. Las estrategias de aprendizaje se adecuaron a la realidad del curso.

22. Las estrategias utilizadas favorecieron los aprendizajes.

23. En la planificación curricular se explicitó el tipo de evaluación que se emplearía.

24. Los conocimientos sobre evaluación que poseía resultaron suficientes para abordar las prácticas.

29. La aplicación de diversos instrumentos de evaluación le demandó mucho tiempo para su preparación.

30. Los instrumentos aplicados para evaluar los aprendizajes fueron efectivos para mostrar los logros.

10. Tuvo la oportunidad de realizar un diagnóstico en Lenguaje y en Matemática al curso que le correspondió trabajar en sus prácticas.

11. La planificación de su trabajo en el aula estuvo precedida de un diagnóstico.

14. Se establecieron metas de aprendizaje precedidas por un diagnóstico del curso y la escuela.

25. Sus conocimientos de evaluación eran distintos a los que poseía el profesor o profesora del curso.

12. Las metas de aprendizaje fueron establecidas, por el profesor o profesora del curso, antes que usted trabajara en sus prácticas.

13. Las metas de aprendizaje para el periodo de prácticas fueron conversadas con el profesor o profesora del curso.

15. En el periodo de observación participante, logró conversar con el profesor o profesora respecto a lo que usted quería lograr en materia de aprendizajes.

4. Sintió seguridad del conocimiento que poseía en Lenguaje y Matemática.

6. En el periodo de observación participante pudo tomar conocimiento de los contenidos que se estaban tratando en el curso

7. El profesor o profesora guía del curso le informó de los contenidos disciplinarios que debía abordar.

9. El profesor o profesora guía del curso, donde realizó las prácticas, le comunicó las metas de aprendizaje para las disciplinas de Lenguaje y Matemática.

19. La escuela requirió de recursos para implementar las estrategias de aprendizaje formuladas por Ud.

20. La escuela le proporcionó los recursos necesarios para llevar a cabo estrategias de aprendizaje.

26. Las evaluaciones fueron planificadas en conjunto con el profesor o profesora del curso.

\begin{tabular}{lllllll} 
F1 & F2 & F3 & F4 & F5 & F6 & F7 \\
\hline
\end{tabular}

5

28. Se aplicaron distintos instrumentos de evaluación para conocer los aprendizajes logrados.

2. Al inicio de sus prácticas recibió información respecto a las metodologías utilizadas, proyectos y programas que se estaban ejecutando en el curso que le correspondió.

21. Los aprendizajes logrados no requirieron de estrategias nuevas.

27. El instrumento más empleado para la evaluación de los aprendizajes fue la prueba escrita. *

A

$r_{\text {i-total corregida }}$

$\%$ de varianza explicada

Número de ítems

\begin{tabular}{|c|c|c|c|c|c|c|}
\hline & & .84 & & & & \\
\hline & & .71 & & & & \\
\hline & & .71 & & & & \\
\hline & .31 & .38 & -.35 & & & \\
\hline & & & .62 & & & \\
\hline & & & .74 & & & \\
\hline & & & .67 & & & \\
\hline .41 & & & & .70 & & \\
\hline & & & .38 & .60 & & \\
\hline & & & .44 & .60 & & \\
\hline & & .42 & & .65 & & \\
\hline & & & & & .69 & \\
\hline .32 & & & & & .60 & \\
\hline-.31 & & .33 & & & .56 & \\
\hline & .52 & & & & .55 & \\
\hline .40 & & & .42 & & & .51 \\
\hline & & & & & & .69 \\
\hline & .44 & & & .40 & & .52 \\
\hline .79 & .73 & .80 & .69 & .63 & .68 & .49 \\
\hline .52 & .52 & .62 & .51 & .42 & .46 & .31 \\
\hline $12.9 \%$ & $9.6 \%$ & $9.4 \%$ & $9.0 \%$ & $7.7 \%$ & $6.5 \%$ & $5.3 \%$ \\
\hline 7 & 4 & 4 & 3 & 4 & 4 & 3 \\
\hline
\end{tabular}




\begin{abstract}
Nota: Se han suprimido las cargas factoriales inferiores a .30 para facilitar su lectura. F1 = Contenidos de las prácticas y aplicación de estrategias de aprendizaje; F2 = Conocimiento y aplicación de instrumentos de evaluación; F3 = Diagnóstico y establecimiento de metas de aprendizaje; F4 = Metas de aprendizajes establecidas en conjunto con el establecimiento (profesor); F5 = Conocimientos de los contenidos disciplinarios (lenguaje y matemática); F6 = Requerimientos de recursos didácticos para el aprendizaje, y F7 = Entrega de información sobre programas existentes. ${ }^{*}$ Se refiere al estudiante en prácticas.
\end{abstract}

\title{
Análisis correlacionales
}

Con las variables latentes obtenidas se llevan a cabo análisis correlacionales para lo que se calcula el coeficiente $\mathrm{r}$ de Pearson. En la Tabla 6 se muestra la matriz de correlaciones bivariadas obtenida. Se observa que entre la mayor parte de las variables se dan correlaciones positivas y estadísticamente significativas, lo que viene a indicar que hay un elevado grado de asociación o de dependencia entre ellas, si bien la dimensión diagnóstico y establecimiento de metas de aprendizaje no se relaciona con los factores contenidos de las prácticas y aplicación de estrategias de aprendizaje, diagnóstico y establecimiento de metas de aprendizaje y conocimientos de los contenidos disciplinarios (lenguaje y matemática). Del mismo modo, el factor conocimiento y aplicación de instrumentos de evaluación no correlaciona con entrega de información sobre programas existentes. Esto indica una independencia entre tales variables latentes, tal vez porque aluden a competencias muy diferentes entre sí.

TABLA 6

Matriz de correlaciones bivariadas entre los factores

\begin{tabular}{lccccccc}
\hline & F1 & F2 & F3 & F4 & F5 & F6 & F7 \\
\hline F1 & 1 & - & - & - & - & - & - \\
F2 & $.42^{* *}$ & 1 & - & - & - & - & - \\
F3 & .14 & .11 & 1 & - & - & - & - \\
F4 & $.46^{* *}$ & $.32^{* *}$ & $.26^{* *}$ & 1 & - & - & - \\
F5 & $.55^{* *}$ & $.26^{* *}$ & .12 & $0.46^{* *}$ & 1 & - & - \\
F6 & $.28^{* *}$ & .08 & $.32^{* *}$ & $0.24^{* *}$ & $.17^{*}$ & 1 & - \\
F7 & $.47^{* *}$ & $.33^{* *}$ & $.19^{*}$ & $0.32^{* *}$ & $.30^{* *}$ & $.24^{* *}$ & 1 \\
\hline
\end{tabular}

\footnotetext{
Nota: ${ }^{*} \mathrm{p}<.05 ;{ }^{* *} \mathrm{p}<.01 . \mathrm{F} 1=$ Contenidos de las prácticas y aplicación de estrategias de aprendizaje; F2 = Conocimiento y aplicación de instrumentos de evaluación; F3 = Diagnóstico y establecimiento de metas de aprendizaje; F4 = Metas de aprendizajes establecidas en conjunto con el establecimiento (profesor); F5 = Conocimientos de los contenidos disciplinarios (Lenguaje y Matemática); F6 = Requerimientos de recursos didácticos para el aprendizaje, y F7 = Entrega de información sobre programas existentes.
}

\section{Discusión}

El objetivo de esta investigación es caracterizar el nivel de aprendizajes logrados en las Prácticas de Lenguaje y Matemática de estudiantes de cuarto año de Pedagogía en Educación General Básica, en atención a las competencias específicas del perfil de egreso de la carrera, según su propia opinión.

En primer lugar, y tras analizar los resultados obtenidos, se puede señalar que las siete competencias específicas del perfil de egreso de la carrera fueron evaluadas a través de los 30 ítems del cuestionario aplicado a estudiantes. Al hacer explícitas las competencias, en este estudio, se coincide con Perrenoud (2010) en el sentido de que en los programas de formación de docentes en que las competencias son subyacentes o referenciales, estas se reducen en la práctica a tenerlas subsumidas como metas e indicadores. 
La concomitancia de los aprendizajes obtenidos en las prácticas de Lenguaje y Matemática por el estudiantado y las competencias específicas del perfil de egreso desde su propia opinión, se derivan de los análisis descriptivos.

\section{Estadísticos descriptivos}

Considerando que solo el $55 \%$ del estudiantado encuestado contestó el ítem 8 ("Los contenidos informados por el profesor o la profesora son los mismos que usted había estudiado en la universidad") se decidió eliminarlo, puesto que este no tenía preponderancia en el estudio ni incidía en la evaluación de los aprendizajes logrados de manera gravitante.

Las acciones que se evalúan son observadas entre A veces y Casi siempre, dado que la media de los ítems es 2.5. En general, los sujetos encuestados se desvían de la media, en promedio, 1.07 unidades de la escala. Respecto a la media, el valor más alto lo obtiene el ítem 6, "En el periodo de observación participante pudo tomar conocimiento de los contenidos que se estaban tratando en el curso", respecto del cual, por término medio, el estudiantado se ubica en 3.2, lo que significa que la mayor parte opina que esta acción se observa con alguna nitidez Casi siempre. Su desviación típica es 1.04, lo que indica que están respondiendo con todas las posibles opciones, sus respuestas no se limitan a elegir la alternativa Casi siempre.

El ítem con la media más baja es el 10, "Tuvo la oportunidad de realizar un diagnóstico en Lenguaje y en Matemática al curso que le correspondió trabajar en sus prácticas”, dado que en promedio se ubica en 1.45, por lo que esta acción es observada Casi nunca o A veces en la mayoría de los casos. A su vez, es el ítem con mayor variabilidad (DT $=1.32$ ), lo cual indica que el estudiantado seleccionó todas las opciones posibles de respuesta, es decir, desde Nunca a Siempre, lo que implica que no existe un patrón de respuesta claramente definido.

El ítem 17, "Las estrategias de aprendizaje diseñadas fueron apropiadas para los resultados que esperaba del curso", presenta la menor dispersión $(\mathrm{DT}=0.78$ ), por lo que es posible indicar que hay poca variabilidad en las respuestas de los sujetos participantes en este ítem, es decir, la mayoría está contestando con la misma opción.

En la mayoría de los ítems ( 25 de 30 ) el coeficiente de asimetría es negativo, lo que significa que los valores tienden a agruparse hacia la derecha de la curva, es decir, por encima de la media. En promedio la curtosis es negativa, lo que indica que la curva es más plana.

\section{Dominio de contenidos disciplinarios (competencias 1 y 4)}

El análisis de frecuencias muestra que el 52.4\% (86 de las 164 personas encuestadas) consideró que Siempre "En el periodo de observación participante pudo tomar conocimiento de los contenidos que se estaban tratando en el curso" (ítem 6), este ítem es el que posee la mayor concentración de respuestas en la alternativa Siempre y Casi siempre. Al respecto, en 2012, el Centro de Perfeccionamiento e Investigaciones Pedagógicas (CPEIP) del Ministerio de Educación entregó los estándares para estudiantado egresado de las carreras de Pedagogía en Educación Básica, en los que especifica que la primera formación de docentes debe preocuparse que sean "profundos conocedores de las disciplinas que enseñarán". En este sentido, el estudio muestra que más del $73 \%$ de estudiantes, antes de comenzar sus prácticas, toma conocimiento de los contenidos disciplinarios que debe enseñar. Asimismo, solo el 1.8\% manifestó nunca observar esta acción.

El estudiantado mayoritariamente marca la opción Siempre o Casi siempre en los ítems que forman parte del factor 1 (véase la Tabla 5), "Contenido de las prácticas y aplicación de estrategias de aprendizaje”, que comprende la primera competencia del perfil de egreso: "Demuestra un dominio de los contenidos 
disciplinarios de la especialidad y en la construcción del saber, en diferentes contextos y situaciones educacionales".

En relación con el inicio de las prácticas y el contacto con la profesora o profesor tutor que evalúa el ítem 7, "El profesor o profesora guía del curso le informó de los contenidos disciplinarios que debía abordar", concentra al $40.9 \%$ de estudiantes en la alternativa de respuesta Siempre, el 33.5\% en la alternativa Casi siempre y solo el $3.7 \%$ consideró Nunca observar esta acción, lo que muestra que reciben información, por parte del personal tutor, previamente sobre los contenidos curriculares que deben abordar durante sus prácticas.

También, en tercer lugar, se corrobora lo señalado precedentemente, cuando se observa que el ítem 1, "Al inicio de sus prácticas recibió información respecto de los contenidos que le correspondía desarrollar en el curso", un 39\% y 31.1\% de estudiantes marcan como una acción observada Siempre y Casi siempre. Mientras que, solo el 4.3\% consideró Nunca observar esta acción. Los ítems 6 y 7 son los que poseen la más alta frecuencia relativa en la alternativa de respuesta Siempre, con un $52.4 \%$ y un $40.9 \%$ respectivamente y viene a confirmar que los ítems que forman parte del factor 1 son coherentes con los estándares de formación docente y con la competencia genérica número uno.

\section{Estrategias de aprendizaje y recursos didácticos (competencias 4 y 5)}

La contribución que las competencias hacen al perfil de egreso se fundamenta, esencialmente, en las estrategias y recursos de aprendizajes que el futuro personal docente es capaz de desarrollar en sus prácticas. De acuerdo con esta categorización, es preciso señalar que a partir de la epistemología se favorece el desarrollo de competencias instrumentales cognitivas y metodológicas al proceso de formación profesional (Fernández, 2013). Desde esta perspectiva, el estudio indica que más de la mitad (51.8\%) de estudiantes consideró que Casi siempre "Las estrategias de aprendizaje diseñadas fueron apropiadas para los resultados que esperaba del curso" (ítem 17). Este resultado está referido solo a la alternativa Casi Siempre. Es interesante señalar que el ítem 17 es el que posee la frecuencia relativa más alta en la mencionada alternativa.

En la alternativa de respuesta Casi siempre, la segunda frecuencia relativa más alta es alcanzada por el ítem 18, "Las estrategias de aprendizaje se adecuaron a la realidad del curso", acción observada por el 44.5\% (73 de 164). Al respecto, Tejada (2011) admite que las "realizaciones profesionales" están vinculadas directamente con la competencia del personal docente para proporcionar "oportunidades de aprendizaje" adaptadas a las características de los individuos o grupos y a la capacidad de orientación contextualizada del proceso de aprendizaje. Luego, la tendencia en las respuestas de estudiantes a este ítem, se corresponde en gran medida con lo señalado por Tejada (2011) y los requerimientos de las competencias del perfil de egreso de la carrera.

En discrepancia con lo anterior, entre las acciones menos observadas destaca que el 34.8\% de estudiantes consideró que Nunca "Tuvo la oportunidad de realizar un diagnóstico en Lenguaje y en Matemática al curso que le correspondió trabajar en sus prácticas” (ítem 10). Además, el 18.9\% expresó la alternativa de respuesta Casi nunca en este ítem, por lo que, de forma conjunta, más de la mitad de estudiantes (53.7\%) Nunca o Casi nunca aplicó un diagnóstico de las asignaturas Lenguaje y Matemática en los cursos en los que llevaron a cabo sus prácticas.

Por otro lado, el 25\% de estudiantes manifestó que Nunca "La planificación de su trabajo en el aula estuvo precedida de un diagnóstico" (ítem 11). A su vez, el 22.6\% consideró Casi nunca observar esta acción. Al sumar ambas alternativas de respuesta, es posible indicar que el $47.6 \%$ consideró que Nunca o Casi nunca observó el ítem 11.

Este resultado produce una divergencia compleja, porque si no es posible para el estudiantado en prácticas realizar un diagnóstico, tampoco es posible adecuar estrategias de aprendizaje. Sin embargo, Le Boterf (2001) plantea que profesional es quien puede manejar una situación compleja y sabe actuar y reaccionar con pertinencia. Asimismo Ávalos y Matus (2010) señalan que, aunque la oportunidad de aprender está 
contenida en el currículo expuesto en el proceso de formación, comprende experiencias de aprendizaje práctico en contextos educacionales escolares, que es lo que muestra este resultado, ya que el grupo participante en este estudio, sin haber realizado un diagnóstico, pudo hacer adecuaciones metodológicas tal y como indica el Marco para la buena enseñanza (MBE, 2003) en el dominio A, "Preparación de la enseñanza", criterio A-2, donde se señala que el profesorado debe conocer las características, conocimientos y experiencias de sus estudiantes, respondiendo de esta manera a la competencia para manejar situaciones complejas.

\section{Metas de aprendizaje, decisiones pedagógicas y actitud critica y proactiva (competencias 2, 6y 7)}

En relación con las metas de aprendizaje, en el ítem 13 del cuestionario, "Las metas de aprendizaje para el periodo de las prácticas fueron conversadas con el profesor o profesora del curso", el $61.6 \%$ de las respuestas se ubican en las alternativas Siempre y Casi siempre. El Marco para la buena enseñanza (MBE, 2003), en el dominio B, "creación de un ambiente propicio para el aprendizaje", criterio B-2, señala que el personal docente debe manifestar altas expectativas y metas sobre las posibilidades de aprendizaje y desarrollo de su alumnado. Si bien las metas de aprendizajes son conversadas entre docentes y estudiantes como indica el último grupo, mayoritariamente, el estudiantado en prácticas no participa en su definición, según sugiere el MBE.

\section{Planificación de los aprendizajes (competencias 2, 4y 5)}

Con respecto a las planificaciones que el futuro personal docente debe elaborar para llevar a cabo su trabajo en el aula, el estudio muestra que estas se realizan, no solo para el desarrollo de los aprendizajes, sino también para las evaluaciones que son parte del proceso de enseñanza y aprendizaje, como indica el ítem 14, "Fue fundamental que el estudiantado planificara sus evaluaciones", que fue observado completamente (Siempre) por el $74.5 \%$, mientras solo el 3.6\% consideró Nunca observar esta acción.

El desarrollo de la formación inicial docente en Chile ha generado un conjunto de exigencias como son la formación por competencias que ha venido a superar al profesorado de la pedagogía tradicional. Asún, Zúñiga y Ayala (2013) señalan que, de una planificación de los aprendizajes “rígida y orientada por contenidos”, el profesorado formado por competencias, como expresa también el perfil de egreso de la carrera, debe pasar a exigencias más flexibles y orientadas hacia las condiciones estudiantiles.

Por su parte, el ítem 21, "Los contenidos se llevaron a cabo tal cual como lo indican los planes y programas de estudio del Ministerio", resalta porque es considerado por el $67.3 \%$ como una acción observada siempre. Considerando conjuntamente las alternativas Siempre y Casi siempre, el porcentaje se eleva a $87.3 \%$. Además, destaca que nadie seleccionó las alternativas de respuesta Nunca o Casi nunca.

Según los resultados del estudio, la totalidad de estudiantes trabaja en sus prácticas con los planes y programa del Ministerio, lo que sorprende, ya que estos tienen carácter solo indicativo y son diseñados centralmente de acuerdo con las bases curriculares vigentes y no consideran ni las singularidades y particularidades de la escuela, ni las de su contexto. Ello indica que el estudiantado copia y aplica planificaciones. Esto se contradice con la competencia específica 1, que apunta a que el futuro personal docente contextualice los contenidos de especialidad, y los integre, de manera armónica, creativa e innovadora, en diferentes escenarios educativos, tanto rurales como urbanos, a través de proyectos y propuestas pedagógicas acordes con el desarrollo local, regional y nacional (Universidad del Biobío, 2009). De este modo, se puede afirmar que los aprendizajes de planificación curricular no se están logrando de la manera establecida por el Marco para la buena enseñanza (MBE, 2003) en el dominio A, criterio A-4: "organiza los objetivos y contenidos de manera coherente con el marco curricular y las particularidades de sus estudiantes". 


\section{Evaluación de los procesos de aprendizaje (competencia 3)}

Todo proceso educativo debe ser evaluado con exigencias pedagógicas complejas, según el modelo de profesorado ideal formado por competencias de Asún et al. (2013) y el Marco para la buena enseñanza (MBE, 2003) que pone el acento en los dominios A y C, específicamente en el dominio C donde establece los criterios de una enseñanza para el aprendizaje de la totalidad de estudiantes. El criterio C-6 indica que el personal docente debe "evaluar y monitorear el proceso de comprensión y apropiación de los contenidos por parte de sus estudiantes”. Claramente la evaluación es constitutiva de los procesos de enseñanza y aprendizaje que docentes y futuro personal pedagogo deben dominar. En este sentido, el estudio muestra que el ítem 25 , "Sus conocimientos de evaluación eran distintos a los que poseía el profesor o profesora del curso", y el ítem 26, "Las evaluaciones fueron planificadas en conjunto con el profesor o profesora del curso", considerando conjuntamente las alternativas Nunca y Casi nunca, son acciones observadas por el 39.1\% y $40.9 \%$, respectivamente.

Sobresalen positivamente los ítems 22, "Las estrategias utilizadas favorecieron los aprendizajes", 7, "El profesor o profesora guía del curso le informó de los contenidos disciplinarios que debía abordar”, 6, "En el periodo de observación participante se pudo tomar conocimiento de los contenidos que se estaban tratando en el curso" y 18, "Las estrategias de aprendizaje se adecuaron a la realidad del curso", porque, al considerar conjuntamente las frecuencias de las alternativas Casi siempre y Siempre, se obtienen porcentajes del 76.3\%, del $74.4 \%$, del $73.7 \%$ y del $72.5 \%$, respectivamente. Esto viene a indicar que son acciones observadas con bastante frecuencia por el estudiantado sometido a estudio.

Las estrategias utilizadas para favorecer los aprendizajes (información de los contenidos disciplinarios, periodo de observación para conocer los contenidos que serán tratados en el curso y la adecuación de las estrategias de aprendizajes a la realidad del curso) expresadas en los ítems $22,7,6$ y 18, de los factores 1 y 5 , que tienen que ver con contenidos de las prácticas y con conocimientos de los contenidos disciplinarios respectivamente, son acciones observadas en las prácticas por estudiantes. Esto es consistente con los principios que tienen mayor consenso en el modelo de formación por competencias de Tobón, Prieto y Fraile (2010), cuando señalan que el profesorado debe orientar sus acciones a formar competencias y no a enseñar contenidos, los cuales deben ser solo medios para el desarrollo de estas.

\section{Conclusiones}

Los resultados del estudio conducen a plantear algunas implicaciones prácticas, que permitirán, a la Carrera de Pedagogía en Educación General Básica, con la información recogida, organizar talleres de prácticas tempranas (séptimo semestre) en las asignaturas de Lenguaje y Matemática, haciendo, antes de las prácticas, un énfasis en los conocimientos pedagógicos de planificación curricular llevada al aula y en la evaluación. También parece necesario introducir en estos talleres actividades de gestión curricular, que le permitan al estudiantado discriminar, por ejemplo, los instrumentos curriculares que son exclusivamente indicativos y los obligatorios para sus planificaciones curriculares.

La investigación indica que el estudiantado aplica planificaciones que otras personas han diseñado y que no conocen los instrumentos de evaluación que deberían emplear según los aprendizajes que se van a evaluar. Con independencia de que la profesora o el profesor tutor determine cómo evaluar a los alumnos y alumnas, el estudiantado en prácticas, en el séptimo semestre de la carrera, deben haber alcanzado aprendizajes pedagógicos sólidos de evaluación para tomar decisiones autónomas en el proceso. Esta acción, de llevarse a cabo, se articula con la mayoría de las competencias del perfil de egreso.

En este mismo sentido, es fundamental que los grupos de estudiantes, previamente, practiquen distintos tipos de planificaciones orientadas a contextualizar las prácticas pedagógicas de acuerdo con el curso que les 
corresponderá atender. Así se podría establecer sinergia entre las prácticas y las competencias del perfil de egreso. Hay que tener en cuenta que las "competencias solo son definibles en la acción" (Tejada y Ruiz, 2016, p. 20).

El estudio tiene relevancia práctica para la carrera, puesto que aporta conocimientos nuevos desde una instancia académica, también nueva, en la formación de docentes y que ocurre fuera de las aulas universitarias, lo que hace necesario extender el periodo denominado "observación participante" con el propósito que el estudiantado llegue a conocer la forma de gestionar el currículo y la realidad del grupo o del curso que le corresponderá atender. Desde esta misma lógica, es recomendable que la Universidad, en general, y la carrera, en particular, instauren "convenios de colaboración mutua" con los establecimientos educacionales que establezcan tiempo de permanencia e información pertinente sobre la trayectoria educativa de la institución escolar, con el objetivo de que el estudiantado pueda realizar diagnósticos y conocer las acciones pedagógicas que se derivan de las clases. Siguiendo esta misma idea, es conveniente que el profesorado tutores sea parte de los análisis de contenidos disciplinarios que se llevarán a sus aulas en lenguaje y matemática, para que se genere lo que Hernández, Martínez, Martínez y Monroy (2009) llaman interacción docente - estudiante, entendida como facilitadora de la adquisición de aprendizajes y de la comprensión del "saber hacer" para el desarrollo de las competencias.

Esta última propuesta se condice plenamente con las prácticas de docentes de las escuelas del estudio, en el sentido de que los contenidos disciplinarios requieren ser analizados previamente en razón de su contextualización y pertinencia.

Las competencias específicas del perfil de egreso se sitúan en los ámbitos cognitivos, afectivos y sociales que el estudiantado debiera adquirir en su periodo de formación y la forma como las prácticas tempranas, en estas dos asignaturas, debieran contribuir al logro de aprendizajes coherentes con estas definiciones; sin embargo, lo que señala el estudio es que el estudiantado en prácticas recibe información por parte de la profesora o profesor tutor respecto de los contenidos que tratarán, pero no hay un trabajo colaborativo desde el punto de vista pedagógico que dé solvencia técnica y pedagógica al trabajo del novel practicante en el aula y en la relación directa con el alumnado, por lo que se considera que la carrera de Pedagogía en Educación General Básica debiera tomar acciones tendentes a producir esta articulación entre practicante y personal tutor, cuyo impacto formativo redundaría en el logro de aprendizajes efectivos.

Es relevante señalar que el estudio pone de manifiesto que los conocimientos, habilidades y actitudes, componentes esenciales de las competencias pedagógicas que el alumnado adquiere en su formación durante las prácticas, se encuentran subsumidos en el periodo, toda vez que las metas de aprendizaje son definidas por la profesora o profesor tutor sin participación estudiantil. Una acción correctiva implicará que la carrera proporcione información a docentes de tutoría respecto de las competencias que el estudiantado debe desarrollar durante las prácticas, desagregando de las competencias específicas las que tienen que ver directamente con el "saber hacer" en el aula. Esto último es coherente con la premisa según la cual las competencias profesionales docentes se abordan con fines claros, socializados, compartidos y asumidos por la institución formadora (Tobón, 2008).

\section{LIMITACIONES DEL ESTUDIO}

Ejerciendo la autocrítica se reconocen algunas limitaciones de este trabajo. Una de ellas tiene que ver con la validez externa, "que se refiere a la capacidad de generalizar los resultados a otros grupos, a otros contextos y a otros momentos temporales" (Ato, López y Benavente, 2013, p. 1039). La procedencia de los sujetos participantes y el procedimiento de muestreo no permiten generalizar los resultados obtenidos. Otra limitación se refiere a la validez de constructo, entendida como la capacidad de definir y operativizar apropiadamente las variables de la investigación (Ato et al., 2013). Una cuestión es el análisis lógico entre competencias, indicadores e ítems; y otra, muy diferente, las variables latentes obtenidas. Ello podría deberse a 
que no se definieron adecuadamente los indicadores al construir el instrumento, lo que supondría reformular algunos ítems. También el procedimiento de autoinforme, que subyace al instrumento empleado, puede hacer posible que los grupos participantes incurrieran en el sesgo de la deseabilidad social. Son estos los aspectos que trataremos de superar en investigaciones futuras.

Ahora que se cuenta con un instrumento para indagar las opiniones de estudiantes sobre los talleres de prácticas, también de cara al futuro, aumentando el tamaño de la muestra, sería conveniente llevar a cabo un análisis factorial confirmatorio, con el fin de valorar la bondad de ajuste del modelo que subyace al cuestionario o complementar los resultados con información cualitativa, siguiendo la estrategia de los grupos focales para ayudar a interpretar los resultados y para clarificar los resultados oscuros de la encuesta siguiendo las propuestas de Morgan (1996). Estos retos quedan pendientes para investigaciones por hacer.

\section{REFERENCIAS}

Asún, R., Zúñiga C., y Ayala, M. (2013). La formación por competencias y los estudiantes: Confluencias y divergencias en la construcción del docente ideal. Calidad en la educación, 38, 277-304. doi: 10.4067/ s0718-45652013000100008

Ato, M., López, J. J., y Benavente, A. (2013). Un sistema de clasificación de los diseños de investigación en psicología. Anales de Psicología, 29(3), 1038-1059. doi: 10.6018/analesps.29.3.178511

Ávalos, B. (2007). El desarrollo profesional continuo de los docentes: Lo que nos dice la experiencia internacional y de la región latinoamericana. Revista Pensamiento Educativo, 41(2), 77-100.

Ávalos, B. y Matus, C. (2010). La formación inicial docente en Chile desde una óptica internacional. Santiago, Chile: Ministerio de Educación.

Castro, J. (2015). Incidencia de los talleres de práctica en la formación inicial docente de la Carrera de Pedagogía en Educación General Básica (Tesis de doctorado inédita). Universidad de Alicante, España.

De Acedo, M. L. S. (2010). Competencias cognitivas en educación superior. Madrid, España: Narcea.

Fernández, A. (2013). Profesionalización docente en la universidad: Implicaciones desde la formación. Revista de la Universidad y Sociedad del Conocimiento, 10, 170-184.

Hernández, F., Martínez, P., Martínez, M. y Monroy, F. (2009). Aprendizaje y competencias: Una nueva mirada. Revista Española de Orientación y Psicopedagogía, 20(3),312-319. Recuperado de http://www2.uned.es/reop/ pdfs/2009/20-3\%20-\%20Fuensanta\%20Hernandez\%20Pina.pdf

Le Boterf, G. (2001). Ingeniería de las competencias. Barcelona, España: Gestión 2000.

MBE. Ministerio de Educación. (2003). Marco para la buena enseñanza. Santiago, Chile: Centro de Perfeccionamiento e Investigaciones Pedagógicas CPEIP.

Ministerio de Educación. (2012) Estándares orientadores para egresados de carreras de pedagogía en educación básica: Estándares pedagógicos y disciplinarios ( $\left.2^{\mathrm{a}} \mathrm{ed}\right)$. Santiago, Chile: Lom Ediciones.

Ministerio de Educación. (2009). Ley general de educación (ley 20.370). Santiago, Chile: Ministerio de Educación. Recuperado de www.mineduc.cl

Ministerio de Educación. (2005). Informe de la Comisión sobre formación inicial docente. Santiago, Chile: Serie Bicentenario.

Morgan, D. (1996). Focus Groups [Grupos de enfoque]. Anual Review of Sociology, 22(1) ,129-152. doi: 10.1146/ annurev.soc.22.1.129

Perrenoud, P. (2010). La formación del profesorado: un compromiso entre visiones inconciliables de la coherencia Revista Interuniversitaria de Formación del Profesorado, 68 (24,2), 103-122. Recuperado de http://aufop.com/ aufop/uploaded_files/articulos/1279237044.pdf

Tejada, J. (2011). Competencias docentes. Profesorado: Revista de Curriculum y Formación del Profesorado, 13(2). Recuperado de http://hdl.handle.net/10481/7373 
Tejada, J. y Ruiz, C. (2016). Evaluación de competencias profesionales en educación superior: Retos e implicaciones. Educación, 19(1), 17-38. doi: 10.5944/educxx1.12175

Tobón, S. (2008). La formación basada en competencias en la educación superior: El enfoque complejo. México: Universidad Autónoma de Guadalajara.

Tobón, S. T., Prieto, J. H. P. y Fraile, J. A. G. (2010). Secuencias didácticas: Aprendizaje y evaluación de competencias. México: Pearson Educación.

Universidad del Bíobío. (2009). Informe n. 22 del proceso de autoevaluación, carrera de pedagogía en educación básica. Chillán, Chile: Universidad del Bío - Bío.

Universidad del Bíobío (2005). Decreto Universitario (N.².459). Chillán, Chile: Universidad del Bío - Bío.

CC BY-NC-ND 